\title{
Correction to: Indole hydrazide compound ZJQ-24 inhibits angiogenesis and induces apoptosis cell death through abrogation of AKT/mTOR pathway in hepatocellular carcinoma
}

Jing Liu, Ying Liu, Jianqiang Zhang, Dan Liu, Yafeng Bao, Tianxing Chen, Tao Tang, Jun Lin, Ying Luo, Yi Jin and Jihong Zhang

Correction to: Cell Death \& Disease https://doi.org/10.1038/s41419-020-03108-2 published online 28 October 2020

The original version of this article unfortunately contained a mistake in Fig. 3A. The correct figure can be found below. The authors apologize for the mistake. The original article has been corrected.

Published online: 25 January 2021 

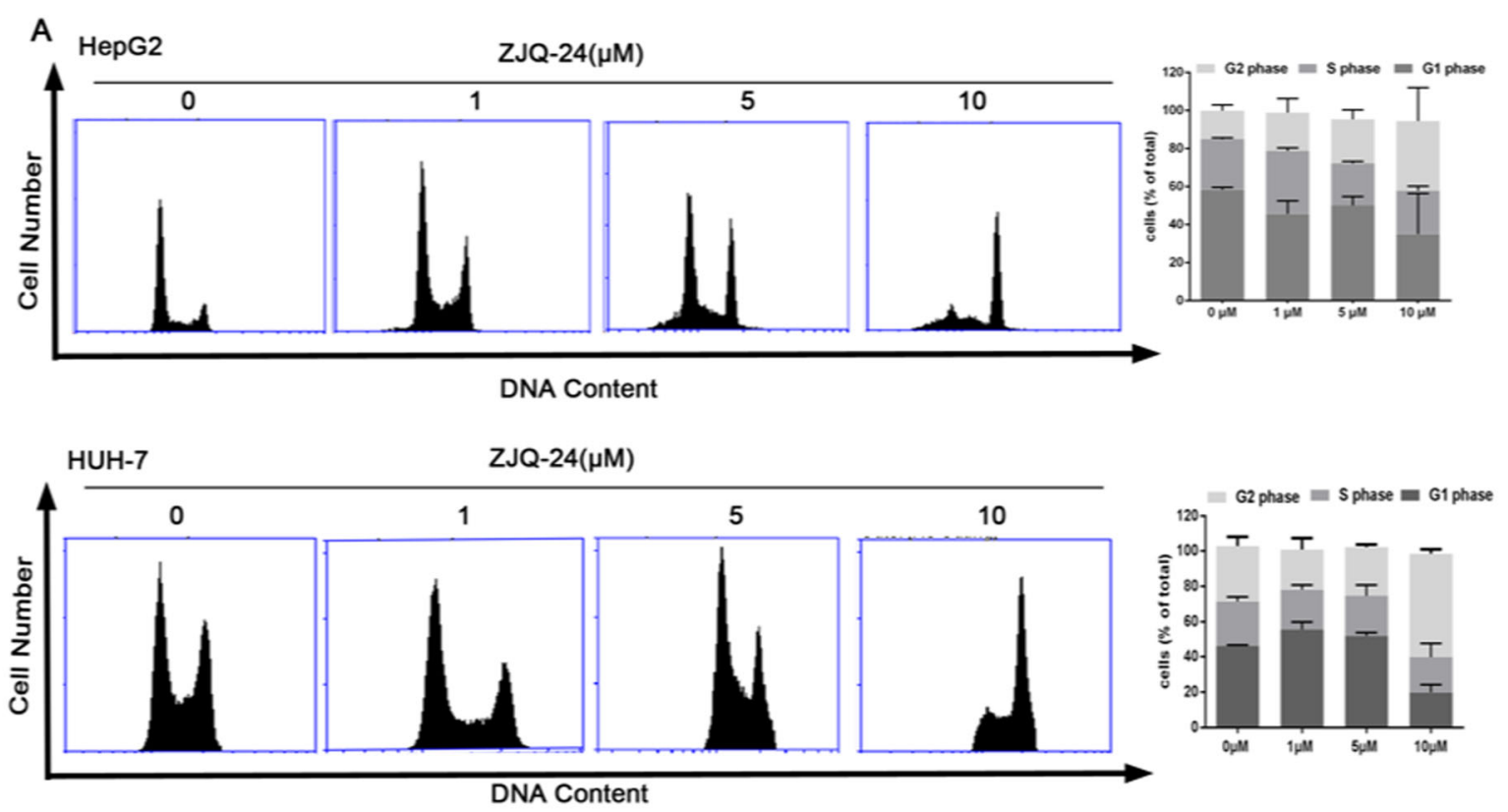

B
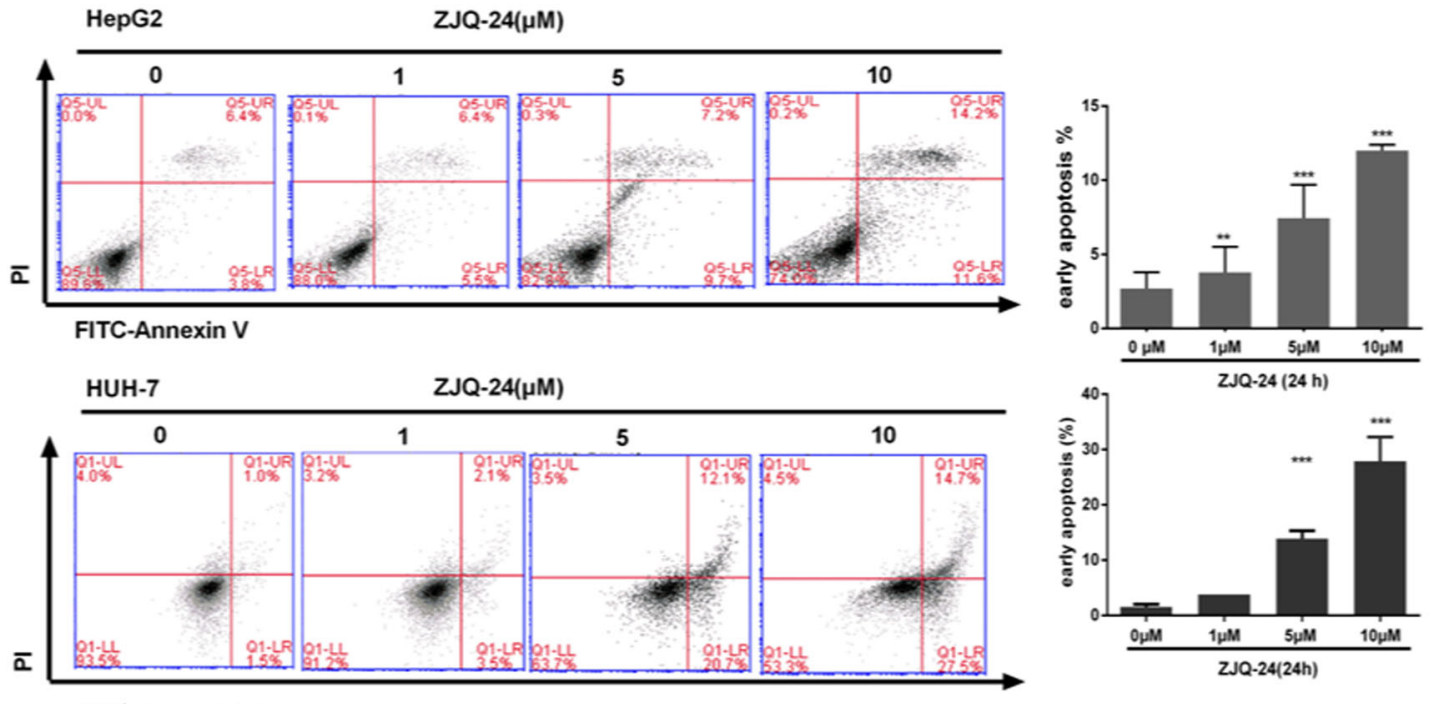

C

ZJQ-24( $\mu M)$

D
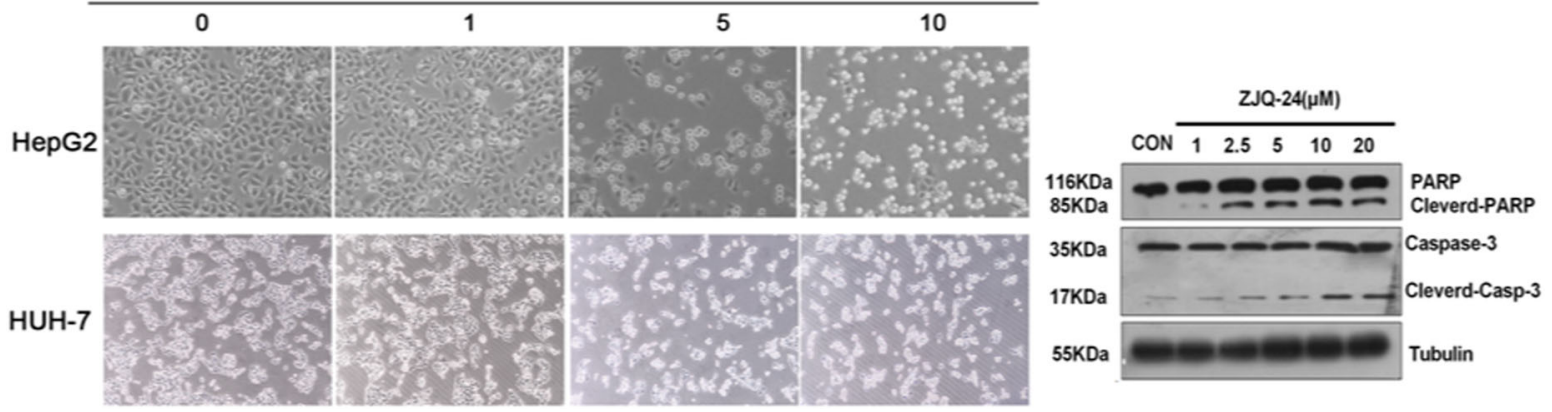

Fig. 3 